\title{
Zinc supports transcription and improves meiotic competence of growing bovine oocytes
}

\author{
Valentina Lodde ${ }^{1}$, Rodrigo Garcia Barros ${ }^{1}$, Priscila Chediek Dall'Acqua ${ }^{2,3}$, Cecilia Dieci ${ }^{1}$, \\ Claude Robert ${ }^{4}$, Alexandre Bastien ${ }^{4}$, Marc-André Sirard ${ }^{4}$, Federica Franciosi ${ }^{1}$ and \\ Alberto Maria Luciano ${ }^{1}$
}

\begin{abstract}
${ }^{1}$ Reproductive and Developmental Biology Laboratory (Redbiolab), Department of Health, Animal Science and Food Safety 'Carlo Cantoni', Università degli Studi di MIlano, Milan, Italy, ${ }^{2}$ Department of Preventive Medicine and Animal Reproduction, School of Agricultural and Veterinarian Sciences, São Paulo State University (UNESP), Jaboticabal, Brazil, ${ }^{3}$ Laboratory of Reproductive Physiology, School of Veterinary Medicine, São Paulo State University (UNESP), Araçatuba, Brazil and ${ }^{4}$ Centre de Recherche en Reproduction, Développement et Santé Intergénérationnelle (CRDSI), Département des Sciences Animales, Université Laval, Québec, Canada
\end{abstract}

Correspondence should be addressed to V Lodde; Email: valentina.lodde@unimi.it

\begin{abstract}
In the last years, many studies focused on the understanding of the possible role of zinc in the control of mammalian oogenesis, mainly on oocyte maturation and fertilization. However, little is known about the role of zinc at earlier stages, when the growing oocyte is actively transcribing molecules that will regulate and sustain subsequent stages of oocyte and embryonic development. In this study, we used the bovine model to gain insights into the possible involvement of zinc in oocyte development. We first mined the EmbryoGENE transcriptomic dataset, which revealed that several zinc transporters and methallothionein are impacted by physiological conditions throughout the final phase of oocyte growth and differentiation. We then observed that zinc supplementation during in vitro culture of growing oocytes is beneficial to the acquisition of meiotic competence when subsequently subjected to standard in vitro maturation. Furthermore, we tested the hypothesis that zinc supplementation might support transcription in growing oocytes. This hypothesis was indirectly confirmed by the experimental evidence that the content of labile zinc in the oocyte decreases when a major drop in transcription occurs in vivo. Accordingly, we observed that zinc sequestration with a zinc chelator rapidly reduced global transcription in growing oocytes, which was reversed by zinc supplementation in the culture medium. Finally, zinc supplementation impacted the chromatin state by reducing the level of global DNA methylation, which is consistent with the increased transcription. In conclusion, our study suggests that altering zinc availability by culture-medium supplementation supports global transcription, ultimately enhancing meiotic competence.

Reproduction (2020) 159 679-691
\end{abstract}

\section{Introduction}

From the time zinc was discovered to be the most abundant transition metal in mouse oocytes (Kim et al. 2010), many studies have focused on the understanding of its possible role in the control of mammalian oogenesis. Thus far, most of the studies considered the function of zinc during oocyte maturation and fertilization. These studies seem to indicate that zinc regulates distinct molecular mechanisms during the two stages, in line with the notion that zinc participates in a vast number of biological processes (Maret 2017)

In the immature fully grown oocyte, zinc is implicated in the maintenance of meiotic arrest (Kong et al. 2012, Tian \& Diaz 2012). Conversely, during maturation, there is a rise in total zinc content, which is key for successful oocyte maturation (Kim et al. 2010, Bernhardt et al.
2011, Tian \& Diaz 2012, Kong et al. 2014, Jeon et al. 2015). In the MII-stage oocyte, the quota of zinc loosely bound to biomolecules in a readily exchangeable form (Outten \& O'Halloran 2001, Dean et al. 2012), referred to as 'labile zinc" or 'free zinc', is accumulated in cortical vesicle-like structures that are released in the extracellular compartment upon fertilization through repetitive exocytic events termed 'zinc sparks', which are in turn implicated in the process of meiotic resumption and block of polyspermy (Kim et al. 2011, Bernhardt et al. 2012, Zhao et al. 2014, Que et al. 2015, 2017, 2019, Duncan et al. 2016). The molecular mechanisms by which zinc regulates all these processes are just starting to be elucidated. However, in reason of the global transcriptional silencing in the fully grown oocytes, they must be transcription independent. 
To date, less is known about the role of zinc at earlier stages of oocyte development, for example, during oocyte growth, when these cells are actively transcribing molecules that will regulate and sustain subsequent stages of oocyte and early embryonic development. The first indication that zinc is fundamental during oocyte growth comes from in vivo studies in the mouse. Specifically, it has been shown that a zinc-restricted diet for 3-5 days before ovulation disrupts oocyte maturation as well as embryonic, fetal and placental development at various levels (Tian \& Diaz 2012, 2013, Tian et al. 2014). Moreover, studies in mouse growing oocytes with uncondensed chromatin in the non-surrounded nucleolus (NSN) configuration have shown that zinc modulation regulates the localization of the metal response element-binding transcription factor-1 (MTF1) (Kong et al. 2014), which is a well-characterized transcription factor that functions as a cellular zinc sensor by coordinating the expression of genes involved in zinc homeostasis (Andrews 2001).

To further advance the knowledge on the role of zinc during oogenesis, we propose the bovine as an experimental model, since the changes that occur within the nucleus during the latest phases of oocyte growth and differentiation before meiotic resumption have been well-characterized (reviewed in Luciano \& Lodde 2013, Luciano et al. 2014b, Bogolyubov 2018). In cattle, as in other mammals, changes in large-scale chromatin configuration in the germinal vesicle (GV) are used as morphological markers of oocyte differentiation (Zuccotti et al. 2005, De La Fuente 2006, Luciano \& Lodde 2013, Luciano et al. 2014a, Bogolyubov 2018). Specifically, four stages of GV oocytes, from GV0 to GV3, have been characterized by increasing level of chromatin compaction (Lodde et al. 2007, 2008, 2009). GV0 oocytes, which typically show uncondensed chromatin and represent the high majority (around $80 \%$ ) of oocytes collected from early antral follicles $(0.5-2 \mathrm{~mm})$, are still growing, meiotically non-competent, transcriptionally active and display low levels of global DNA methylation. In contrast, oocytes with increasing levels of compaction (GV1, GV2 and GV3) are considered 'fully grown' and are typically collected from medium antral follicles, 2-8 $\mathrm{mm}$ in diameter. GV1, GV2 and GV3 oocytes are meiotically competent, while they differ in the ability to form blastocyst after in vitro fertilization, with GV1 oocytes being less competent than GV2 and GV3. Notably, a major drop in transcription occurs during the transition from GV0 to GV1 configuration, similar to the mouse non-surrounded nucleolus/surrounded nucleolus (NSN/SN) transition, also described in humans and other mammalian species (reviewed in Luciano \& Lodde 2013). GV0 to GV1 transition also entails increased levels of global DNA methylation (Lodde et al. 2009).

The bovine model offers the additional following advantages for the purpose of the proposed study. First, a system for the in vitro culture of oocyte, termed IVCO, is available in cattle (Luciano et al. 2011). Thanks to the use of physiological doses of FSH and PDE3 inhibitors, this system is able to promote the crosstalk between oocytes and cumulus cells and, in turn, the oocyte growth and the increase of their meiotic competence when they are further subjected to standard in vitro maturation (IVM) condition (Luciano et al. 2011). Secondly, the transcriptomic profiles of bovine oocytes and surrounding cumulus cells at different stages of differentiation are available. Specifically, they were assessed by microarray analysis (Labrecque et al. 2015, 2016, Dieci et al. 2016) within the EmbryoGENE program (http://emb-bioinfo.fsaa.ulaval.ca/Home/index. html) that has been implemented by collecting and storing transcriptomic data of bovine oocytes, cumulus and granulosa cells under different physiological conditions using the same transcriptomic platform and bioinformatic tools. After being analyzed and validated individually in their respective publication, all the collected transcriptomic data sets were subjected to meta-analysis as described in Khan et al. (2016) and made publicly available through an interactive web interface called EmbryoGENE profiler, which thus provides dynamic expression profiles of any gene of interest in the selected sample (http://emb-bioinfo.fsaa. ulaval.ca/IMAGE/index.html).

Given the previously mentioned observation, we have used the bovine growing oocyte as the experimental model to assess the extent to which zinc plays a role during the latest phase of oocyte growth and differentiation and particularly to the hypothesis that zinc participates in the control of transcription at this stage.

\section{Materials and methods}

All chemicals and reagents were purchased from SigmaAldrich S.r.l.f, unless otherwise stated.

\section{EmbryoGENE dataset mining}

The expression profile of genes encoding for proteins involved in intracellular zinc homeostasis (Kimura \& Kambe 2016) was assessed by mining the publicly available EmbryoGENE profiler (http://emb-bioinfo.fsaa.ulaval.ca/IMAGE/index.html), which contains profiles of microarray expression data from a variety of tissues and conditions, collected by the researchers within the EmbryoGENE network (http://emb-bioinfo.fsaa.ulaval.ca (Khan et al. 2016)). Specifically, the EmbryoGENE profiler was mined to assess the expression profiles of: (1.) members of the solute carrier family 39 A (SLC39A), which encode for Zrt- and Irt-like proteins (ZIP); (2.) members of the solute carrier family $30 \mathrm{~A}$ (SLC30A), which encode for Zn transporters (ZnT) and (3.) members of the protein coding gene metallothioneins (MT). Three sets of previously published microarray data that cover the period of oocyte growth and acquisition of meiotic and developmental competence were considered: (1.) GEO series 
accession number GSE48283: oocytes collected from follicle of different size $(<3,3-5,>5-8$ and $>8 \mathrm{~mm}$ ) (Labrecque et al. 2016); (2.) GEO series accession number GSE48376: oocytes with different degree of chromatin compaction (GV0, GV1, GV2 and GV3) (Labrecque et al. 2015) and (3) GEO series accession number GSE79886: cumulus cells isolated from oocytes with different degrees of chromatin compaction (GV0, GV1, GV2 and GV3) (Dieci et al. 2016). Importantly, each data set was validated by quantitative PCR as reported in each respective publication.

\section{Oocytes collection and culture}

Cumulus-oocytes complexes (COC) were collected and cultured as previously described (Lodde et al. 2007, Luciano et al. 2011). Briefly, bovine (Holstein Friesians) ovaries were harvested at a local abattoir (IT 2270M CE; Inalca S.p.A., Ospedaletto Lodigiano, LO, Italy) from pubertal females subjected to routine veterinary inspection and in accordance to the specific health requirements stated in Council Directive 89/556/ECC and its subsequent modifications. The ovaries were transported to the laboratory within $2 \mathrm{~h}$ in sterile saline solution maintained at $26^{\circ} \mathrm{C}$. All the subsequent procedures, unless otherwise specified, were performed between $35^{\circ} \mathrm{C}$ and $38^{\circ} \mathrm{C}$. COCs were retrieved from 2 to $8 \mathrm{~mm}$ middle antral follicles with a 19-gauge needle connected to an aspiration pump (COOKIVF, Brisbane QLD, Australia) with a vacuum pressure of 28 $\mathrm{mm} / \mathrm{Hg}$. After aspiration, small pieces of ovarian cortex were sliced and examined under a dissecting microscope. COCs were isolated from early antral follicles (0.5-2 mm diameter) by rupturing the follicle wall with a 21-gauge needle. COCs were washed in Medium 199 supplemented with HEPES 20 mM, 1790 units/L Heparin and 0.4\% of BSA (M199-D) and examined under a stereomicroscope. COCs from early antral follicles were collected within $2 \mathrm{~h}$. Only compact COCs with five or more complete layers of cumulus cells and a finely granulated homogenous ooplasm were used. The population of oocytes collected from this follicular class typically contain around $80 \%$ of GV0 stage oocytes and their mean diameter is $110 \mu \mathrm{m}$ (Lodde et al. 2007, Luciano et al. 2011).

The in vitro culture of growing oocyte (IVCO) was performed as previously described with minor modification (Luciano et al. 2011). Oocytes were always cultured in the presence of cumulus cells as COCs. COCs were cultured in $500 \mu \mathrm{L}$ of Medium 199 with $25 \mathrm{mM} \mathrm{NaHCO}_{3}$ and further supplemented with $0.4 \%$ fatty acid-free BSA, $0.68 \mathrm{mM} \mathrm{L-glutamine,} 0.2 \mathrm{mM}$ sodium pyruvate, $0.1 \mathrm{mM}$ cysteamine, $50 \mu \mathrm{g} / \mathrm{mL}$ of kanamycin, $10^{4} \mathrm{IU} / \mathrm{mL}$ of recombinant human follicle-stimulating hormone (r-hFSH; Gonal F; Serono S.p.A.) and $10 \mu \mathrm{M}$ cilostamide, for $24 \mathrm{~h}$ or for different time intervals according to the experimental design (see subsequent section), in four-well dishes (Nunc, Thermo Fisher Scientific) at $38.5^{\circ} \mathrm{C}$ under $5 \%$ $\mathrm{CO}_{2}$ in humidified air. According to the experimental design, IVCO medium was supplemented with $6.6 \mu \mathrm{g} / \mathrm{mL}$ zinc sulfate, which corresponds to $1.5 \mu \mathrm{g} / \mu \mathrm{L}$ of $\mathrm{Zn}^{2+}$. This concentration is within the range of follicular fluid content and has been proved to be beneficial during bovine in vitro maturation (Picco et al. 2010, Anchordoquy et al. 2014). In a preliminary set of experiments, the IVCO medium was supplemented with
$10 \mu \mathrm{M} \quad \mathrm{N}, N, N^{\prime}, N^{\prime}$-tetrakis (2-pyridinylmethyl)-1,2ethanediamine (TPEN), a cell permeable zinc chelator (Kim et al. 2010). To assess the effect of zinc modulation on meiotic competence, after IVCO, COCs were washed in M199-D and cultured for $24 \mathrm{~h}$ under standard serum-free IVM conditions. IVM medium was M199 supplemented with $0.68 \mathrm{mM}$ L-glutamine, $25 \mathrm{mM} \mathrm{NaHCO}, 0.4 \%$ fatty acid-free BSA, $0.2 \mathrm{mM}$ sodium pyruvate, $0.1 \mathrm{mM}$ cysteamine, $50 \mu \mathrm{g} / \mathrm{mL}$ of kanamycin and $0.1 \mathrm{IU} / \mathrm{mL}$ of r-hFSH (Luciano et al. 2011). To assess meiotic progression, at the end of IVM, cumulus cells were mechanically removed and oocytes were fixed in $4 \%$ paraformaldehyde in PBS for $30 \mathrm{~min}$ at room temperature, stained with DAPI and analyzed by fluorescence microscopy to assess meiotic progression. Oocytes from the GV breakdown to the metaphase I stage were classified as intermediate; oocytes at the anaphase I, telophase I and metaphase II stages as mature and oocytes that could not be identified as being at any of the previous stages as degenerate (Luciano et al. 2011).

\section{Assessment of labile zinc content in the oocytes}

Labile zinc content in oocytes with different degree of chromatin compaction at the time of collection was assessed using the cell-permeant zinc indicator FluoZin ${ }^{\mathrm{TM}} 3$-AM dye (Molecular Probes, Termo Fisher Scientific), which had previously been used for spatial and temporal resolution of labile zinc concentrations in both mouse and bovine oocytes (Kim et al. 2011, Lisle et al. 2013, Que et al. 2019). Further validation of the FluoZin ${ }^{\text {TM } 3-A M ~ u s i n g ~ t h e ~ z i n c ~ c h e l a t o r ~ T P E N ~ i s ~ r e p o r t e d ~ i n ~}$ Supplementary Fig. 1 (see section on Supplementary materials given at the end of this article). After collection, COCs from early and medium antral follicles were incubated in $2 \mu \mathrm{M}$ of FluoZin-3 and mixed to an equal volume of Pluronic ${ }^{\circledR}$ F-127 $20 \%$ solution in DMSO (Invitrogen, Molecular Probes, Thermo Fisher Scientific) dissolved in $500 \mu \mathrm{L}$ of M199 with $25 \mathrm{mM}$ $\mathrm{NaHCO}_{3}$ and supplemented with $0.68 \mathrm{mM}$ L-glutamine, $0.4 \%$ fatty acid-free BSA, $0.2 \mathrm{mM}$ sodium pyruvate, $0.1 \mathrm{mM}$ cysteamine, $50 \mu \mathrm{g} / \mathrm{mL}$ kanamycin and $0.5 \mathrm{mM} \mathrm{IBMX}$, in 4-well dishes at $38.5^{\circ} \mathrm{C}$ under $5 \% \mathrm{CO}_{2}$ in humidified air for $90 \mathrm{~min}$. After 1 -h incubation, $1 \mu \mathrm{g} / \mathrm{mL}$ of Hoechst 33342 was added to each well. Subsequently, COCs were denuded mechanically by pipetting to remove cumulus cells in M199D supplemented with IBMX and $1 \mu \mathrm{g} / \mathrm{mL}$ of Hoechst 33342 . Oocytes were placed on slides in $10-\mu \mathrm{L}$ drops of the same medium and covered with a coverslip using secure-seal spacer (Invitrogen, Thermo Fisher Scientific). Digital images of each of the samples were immediately acquired using an epifluorescence microscope (Nikon Eclipse E600, Nikon) equipped with a digital camera (Nikon DS-F12), maintaining identical acquisition settings for all samples. All the images were acquired at the focal plane in which the chromatin was more focused. The oocyte labile zinc content was estimated by assessing the mean fluorescent intensity value emitted by the probe using ImageJ software $(1.49 v)$. The total fluorescent intensity emitted by the oocyte area (integrated density) was subtracted by the background and then normalized by the oocyte area. Mean fluorescent intensity values were $\log _{2}$ transformed. GV stages were classified according to the degree of chromatin compaction, as previously described (Lodde et al. 2007). The GV0 stage 
is characterized by a diffuse filamentous pattern of chromatin in the whole nuclear area; the GV1 stage shows a few points of chromatin condensation; the GV2 stage has the chromatin condensed in distinct clumps; the GV3 stage shows the highest level of compaction, with chromatin organized in a single clump within the nuclear envelope (Luciano \& Lodde 2013).

\section{Assessment of global transcriptional activity}

Transcription was assessed in growing oocytes cultured as COCs in IVCO medium as described previously, in the presence or absence of zinc sulfate for $17 \mathrm{~h}$. In a second set of experiments, transcription was evaluated in oocytes treated with $10 \mu \mathrm{M}$ zinc chelator TPEN, in the absence or presence of zinc sulfate for $5 \mathrm{~h}$ to prove TPEN specificity as previously described (Kim et al. 2010). Global transcriptional activity was evaluated using the Click-iTß RNA Imaging Kit (Invitrogen, Thermo Fisher Scientific), as previously described in mice (Sanchez et al. 2015) and following the manufacture's guide.

Briefly, after treatment, COCs were incubated with $2 \mathrm{mM}$ 5-ethynyl uridine (EU) diluted in IVCO medium for $1 \mathrm{~h}$ at $38.5^{\circ} \mathrm{C}$ under $5 \% \mathrm{CO}_{2}$. Note that zinc and/or TPEN were included in the medium during EU incorporation, according to the experimental design. After EU incorporation, cumulus cells were mechanically removed, oocytes were briefly washed in warm PBS/PVA, fixed in $4 \%$ paraformaldehyde in PBS for $30 \mathrm{~min}$ at room temperature and washed again in PBS/PVA. For detection of EU incorporation into nascent RNA, samples were permeabilized in $0.5 \%$ Triton-X 100 in PBS for $15 \mathrm{~min}$ at room temperature, briefly washed in PBS/ PVA and incubated in Click-iT® reaction cocktail for 30 min at RT protected from light, further washed once in Click-iT ${ }^{\circledR}$ reaction rinse buffer and once more time in PBS/PVA. Oocytes were finally mounted and stained in mounting media, Vectashield antifade mounting media, with DAPI (Vector, Burlingame, CA, USA), using double-sticky tape between the slide and the cover glass. Samples were analyzed and imaged under an epifluorescence microscope as described previously, maintaining identical acquisition settings for all the samples. Chromatin configuration was evaluated under the fluorescence microscope. The GV stage was classified according to the degree of chromatin compaction, as previously described (Lodde et al. 2007). The level of global transcriptional activity was estimated by assessing the mean fluorescent intensity value emitted by the incorporated EU using NIH Image software (1.49v) (Schneider et al. 2012). The mean fluorescent intensity emitted by the nuclear area was subtracted by the mean background intensity of the oocyte's cytoplasm. Note that transcription was quantified only in oocytes that retained a GV0 configuration during culture.

\section{Assessment of global DNA methylation}

Levels of global DNA methylation were assessed by indirect immunofluorescence, as previously described (Lodde et al. 2009), in oocytes subjected to IVCO for $17 \mathrm{~h}$ in the presence or absence of zinc sulfate. Briefly, after culture as COC, the oocytes were freed of cumulus cells and the zona pellucida was digested with $0.5 \%$ of pronase. Samples were then fixed in $4 \%$ paraformaldehyde in PBS for $1 \mathrm{~h}$ at $4{ }^{\circ} \mathrm{C}$, washed in $0.05 \%$ Tween 20 in PBS, permeabilized with $0.2 \%$ Triton $\mathrm{X} 100,0.05 \%$ Tween 20 in PBS for $30 \mathrm{~min}$ at room temperature, treated in $2 \mathrm{M} \mathrm{HCl}$ for $30 \mathrm{~min}$ at room temperature to obtain DNA denaturation and then neutralized in $100 \mathrm{mM}$ Tris $\mathrm{HCl}$ buffer (pH 8.5) for 10 min. Non-specific binding was blocked by incubating the samples in $20 \%$ normal donkey serum, $1 \%$ BSA in PBS for $1 \mathrm{~h}$ at room temperature before primary antibody incubation. The samples were incubated overnight at $4{ }^{\circ} \mathrm{C}$ with a mouse anti-5-methylcytosine (Eurogentec SA, Seraing Belgium) 1:500 in PBS containing 1\% BSA and 0.05\% Tween 20. Samples were extensively washed in 0.05\% Tween 20 in PBS and incubated for $1 \mathrm{~h}$ at RT with a donkey antimouse conjugated with Alexa Fluor 488 (Molecular Probes, Thermo Fisher Scientific, 1:500) in PBS containing 1\% BSA and $0.05 \%$ Tween 20. Samples were mounted in Vecta Shield supplemented with DAPI, incubated for $5 \mathrm{~min}$ at room temperature and immediately observed under epifluorescence microscopy. Images were captured under the same exposure conditions. The level of global DNA methylation was estimated by assessing the mean fluorescent intensity value emitted by the incorporated EU using NIH ImageJ software (1.49v) (Schneider et al. 2012). The mean fluorescent intensity emitted by the nuclear area was subtracted by the mean background intensity of the oocyte's cytoplasm. Note that DNA methylation was quantified only in oocytes that retained a GVO configuration.

\section{Statistical analysis}

Experiments were performed as three independent experiments, unless otherwise specified. Statistical analysis was performed using GraphPad Prism software (GraphPad Prism v. 8). Data were pooled and expressed as mean \pm S.E.M. Data were first analyzed by $\mathrm{D}^{\prime}$ Agostino and Pearson test, in order to assess whether data distribution was normal. Parametric tests (Student's t-test and one-way ANOVA followed by Tukey's multiple comparison test) were used to determine differences when data were normally distributed. A non-parametric test (Mann-Whitney test) was chosen when the data were not normally distributed or assumption on distribution could not be made. In the experiments where the labile zinc content was evaluated, values were $\log _{2}$ transformed. Details on the statistical analysis are indicated in the figure caption. $P<0.05$ were considered statistically significant and indicated as *. Further differences, $P<0.01, P<0.001$ and $P<0.0001$ are noted as $* *, * * *$ and $* * * *$, respectively.

\section{Results}

\section{Zinc transporters and metallothionein's encoding mRNAs are differentially expressed during oocyte growth and differentiation}

To gain insights into the possible role of zinc during oocyte growth and differentiation that occur within the antral follicle before meiotic resumption, we mined the EmbryoGENE profiler to assess the expression profiles of genes that encode for proteins that modulate zinc homeostasis in mammalian cells (Kimura \& Kambe 2016, 
Maret 2017). Specifically, two major classes of ion transporters participate in this process: the SLC39A family members, which encode for ZIP proteins that transport zinc into the cytosol from either the extracellular space or intracellular stores such as the endoplasmic reticulum, and the SLC3OA family members, which encode for the $\mathrm{ZnT}$ proteins that mediate zinc efflux from the cytosol (Kimura \& Kambe 2016). Moreover, MTs bind metals intracellularly, thus lowering their concentration at critical sites, or function as buffering molecules providing labile zinc for use by target proteins or enzymes when zinc is limited (Kimura \& Kambe 2016). Thus, the EmbryoGene profiler was mined to assess the expression profile of the known members of the SLC39A, SLC30A and $M T$ gene families in oocytes collected from follicle of different size (Labrecque et al. 2016) as well as in oocytes with different degree of chromatin compaction and in their surrounding cumulus cells (Labrecque et al. 2015, Dieci et al. 2016).

As summarized in Table 1, the expression profiles of 21,14 and 6 probes (representing constitutive, alternative 3'UTR and splicing variants) of the SLC39A, SLC30A and MT gene families were considered, respectively. Of these, several transcripts were differentially expressed in oocytes (Fig. 1A and B). Specifically, SLC39A7/ZIP7, SLC39A8/ ZIP8, SLC39A10/ZIP10 (both constitutive and alternative 3'UTR forms), SLC39A12/ZIP12, SLC39A14/ZIP14 (alternative 3'UTR), SLC30A1/ZnT1 and SLC30A5/ZnT5 were differentially expressed according to follicle size and the degree of chromatin compaction. Furthermore SLC39A6/ZIP6, SLC39A10/ZIP10 (Alternative 3'UTR) and $S L C 30 A 9 / Z n T 9$ were differentially expressed uniquely in oocytes derived from follicle of different size (Fig. 1A), while SLC39A9/ZIP9 (alternative 3'UTR), SLC30A3/ZnT3, SLC30A6/ZnT6 and SLC30A7/ZnT7 were differentially expressed uniquely in oocytes with different degree of chromatin compaction (Fig. 1B). A lower number of genes, namely SLC39A7/ZIP7, SLC39A8/ZIP8, SLC39A14/ZnT14 alternative 3'UTR and $S L C 30 A 4 / Z n T 4$, were differentially expressed in cumulus cells according to chromatin configuration (Fig. 1C).

\section{Zinc supplementation improves the meiotic competence of growing oocytes}

Unlike fully grown oocytes isolated from middle antral follicles, bovine oocytes collected from early antral follicles are not capable of spontaneously resuming meiosis once isolated from the follicular compartment and progressing to the MII stage. However, their ability to undergo meiosis and early embryonic development is, at least partially, enhanced when oocytes are cultured for $24 \mathrm{~h}$ before in vitro maturation in a low FSH concentration-based culture system that sustains oocytecumulus cells coupling (Luciano et al. 2011). To the best of our knowledge, this is one of the few available culture systems for this class of oocytes. In the present study, we used this culture system as an experimental model to further assess the role of zinc during oogenesis. Preliminary experiments showed a detrimental/toxic effect of long-term treatment with the zinc chelator TPEN, as none of the oocytes exposed to IVCO supplemented with TPEN for $24 \mathrm{~h}$ were able to progress through meiosis when further subjected to standard IVM and instead appeared degenerated. Therefore, we focused our attention on the effects of supplementing the IVCO medium with zinc and evaluating the effect on meiotic progression after standard IVM.

As shown in Fig. 2, zinc supplementation induced a significantly higher percentage of mature oocytes and a lower percentage of oocytes at intermediated stages of meiotic progression, when compared to the control group. No differences were observed in the percentage of oocytes that did not resume meiosis nor in the degenerated class.

\section{Labile zinc content decreases during physiological transcriptional silencing}

The increased meiotic competence in response to zinc supplementation during IVCO suggests that zinc supports some of the processes that play a role in oocyte competence acquisition. Typically, the high majority (around 80\%) of growing oocytes have their chromatin organized in a GVO configuration and are transcriptionally active (Fair et al. 1995, 1996, Lodde et al. 2007, 2008, Luciano et al. 2011). Therefore, we hypothesized that zinc supplementation might support transcription in GV0 oocytes. We tested this hypothesis using two experimental approaches.

First, we evaluated the labile zinc content in oocytes with different degrees of chromatin compaction at the time of isolation from early and middle antral follicles, since a major transcriptional silencing occurs during the transition from GV0 to GV1 stages and GV1, GV2 and GV3 oocytes are typically collected from middle antral follicles (Lodde et al. 2007, 2008). As shown in Fig. 3, quantification of the FluoZin ${ }^{\mathrm{TM}} 3-\mathrm{AM}$ dye signal fluorescent intensity revealed a significant decrease of labile zinc content during the transition from GV0 to GV1 oocytes. Labile zinc remained low in GV2 oocytes, while tended to increase again in GV3 oocytes (Fig. 3B).

\section{Modulation of zinc availability affects global transcriptional activity}

To more directly evaluate the role of zinc in the modulation of oocyte's transcription, in a second set of experiments, we supplemented the IVCO medium with zinc and/or with the zinc chelator TPEN and assessed global transcription in GV0 oocytes by means of EU incorporation into nascent RNA. As shown in Fig. 4A, this method clearly allowed the detection of RNA transcription in bovine oocytes. At the time of 


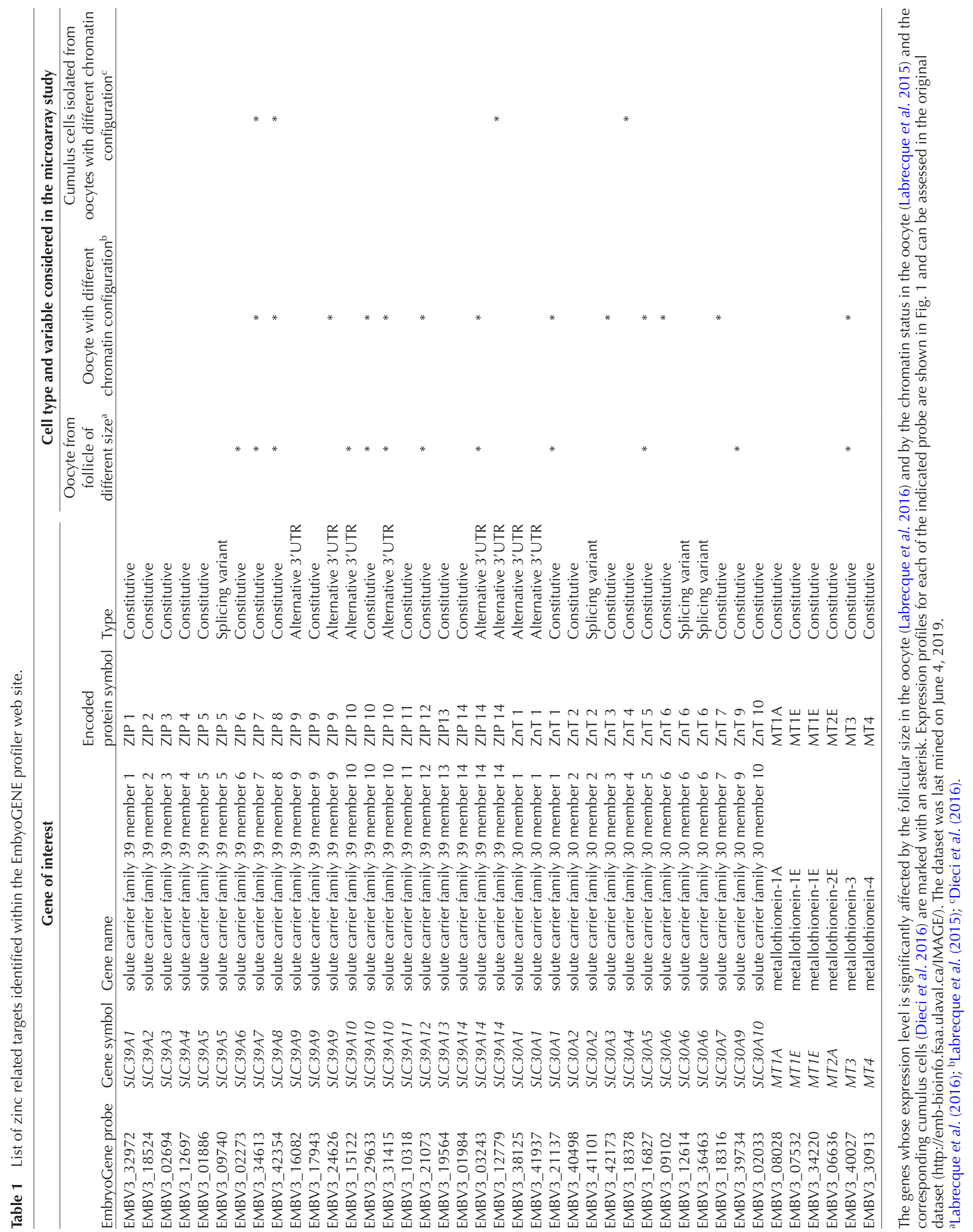




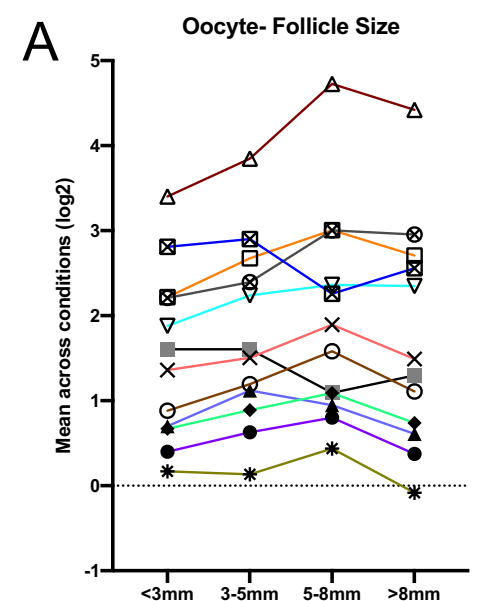

- SLC39A6

- SLC39A7

^ SLC39A8

○ SLC39A10

- SLC39A10 alt 3'UTR

曰 SLC39A10 alt 3'UTR

$\triangle$ SLC39A12

$\nabla$ SLC39A14 alt 3'UTR

* SLC30A1

$\times$ SlC30A5

SLC30A9

МT3

B

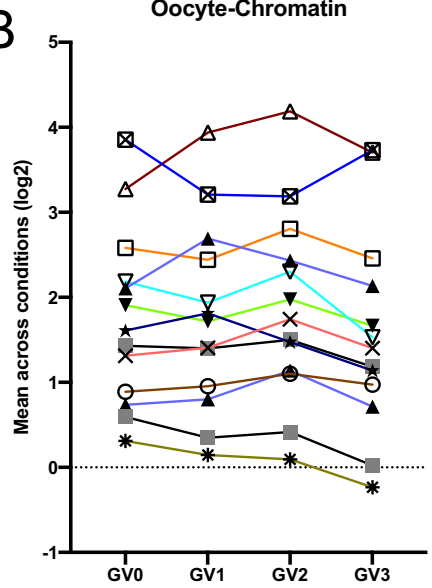

- SLC39A7

^ SLC39A8

$\checkmark$ SLC39A9 alt 3'UTR

○ SLC39A10

曰 SLC39A10 alt 3'UTR

$\triangle$ SLC39A12

$\nabla$ SLC39A14 alt 3'UTR

* SLC30A1

* SLC30A3

$\times$ SlC30A5

- SLC30A6

^ SLC30A7

\MT3

-2.- SLC39A7

- SLC39A8

$\diamond$ SLC39A14 alt 3'UTR

+ SLC30A4

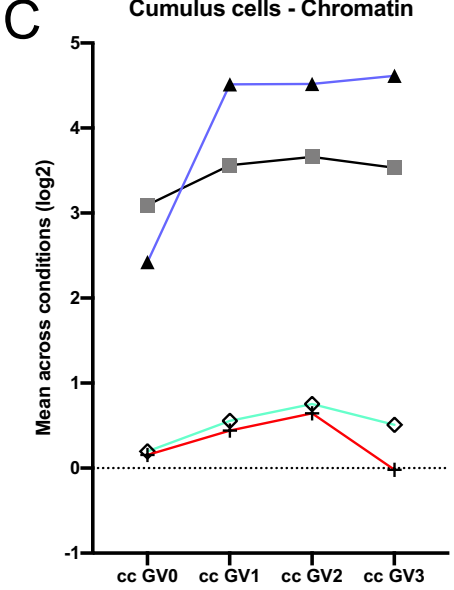

Figure 1 Levels of differentially expressed mRNAs encoding zinc transporters (ZIP and ZnT) and metallothioneins (MT) during oocyte growth and differentiation. Graphs showing changes of mRNA levels encoding the differentially expressed members of the SLC39A,

SLC30A and MT gene families in oocytes collected from follicles of different sizes (A) and in oocytes and corresponding cumulus cells with different degrees of chromatin compaction (B and C). The data represent the profiles in the original microarray analysis and are obtained/imported from the EmbryoGENE profiler website (http:// emb-bioinfo.fsaa.ulaval.ca/IMAGE/) on June 4, 2019. Data were generated by Labrecque et al. $(2015,2016)$ and Dieci et al. (2016).

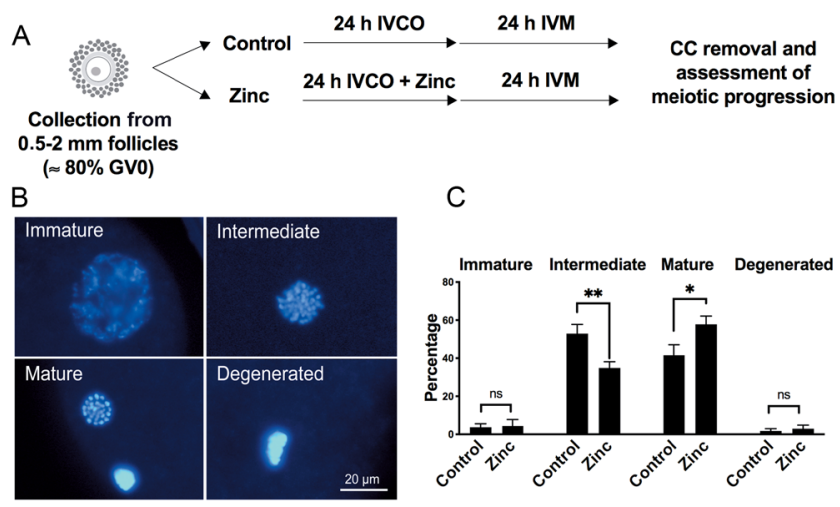

Figure 2 Effect of zinc supplementation during IVCO on meiotic competence acquisition. (A) Schematic representation of the experimental design. (B) Representative epifluorescent images showing immature $(\mathrm{GV})$, intermediate $(\mathrm{MI})$, mature (MII, polar view and first polar body) and degenerated oocytes as assessed by DNA staining with DAPI. (C) Graphs showing the percentage of immature, intermediate, mature and degenerated oocytes in the control (IVCO in the absence of zinc followed by standard IVM) and zinc (IVCO in the presence of zinc followed by standard IVM) groups. Data were analyzed by Mann-Whitney test. Values are means \pm S.E.M. $(n=6)$. * and $* *$ indicate significant differences between groups $\left({ }^{*} P<0.05\right.$; $* * P<0.01)$. A total of 98 and 102 oocytes were analyzed for control and zinc groups, respectively, in three independent experiments with two technical replicates each.

isolation, a positive signal was detectable only in GV0 oocytes and not in the negative control or fully grown GV1 oocytes. Zinc supplementation for $17 \mathrm{~h}$ did not change the distribution of GV stages, and the percentage

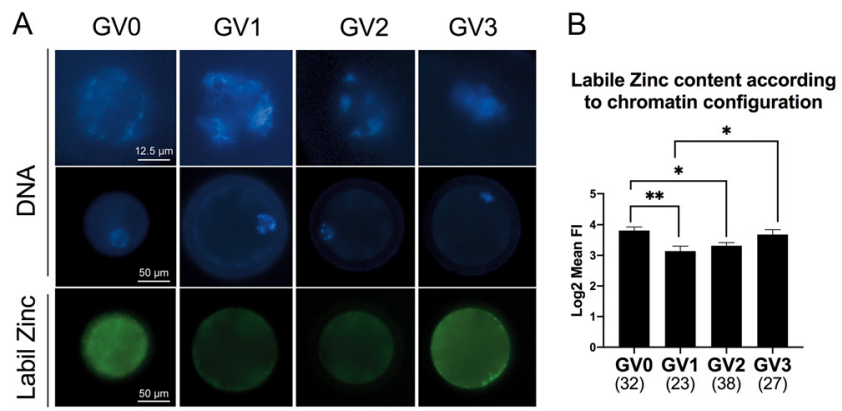

Figure 3 Relationship between large-scale chromatin configuration of the oocyte and labile zinc content. (A) Representative images showing chromatin staining by Hoechst33342 (upper and middle panels) and labile zinc staining by Fluo Zin (lower panel) in oocytes with GV0, GV1, GV2 and GV3 chromatin configuration. All images were captured at the GV focal plane. (B) Graph showing quantification of labile zinc content as assessed by Fluo Zin loading ad fluorescent image analysis in growing oocytes collected from small antral follicle with a GV0 chromatin configuration and fully grown oocytes collected from middle antral follicle with GV1, GV2 and GV3 chromatin configuration as assessed by Hoechst 33342 staining. Total numbers of oocytes analyzed are shown in brackets. Data were pooled together and analyzed by one-way ANOVA followed by Tukey's multiple comparison test. Values are means \pm S.E.M. ${ }^{*}$ and ${ }^{* *}$ indicate significant differences between groups $(* P<0.05 ; * * P<0.01)$. 

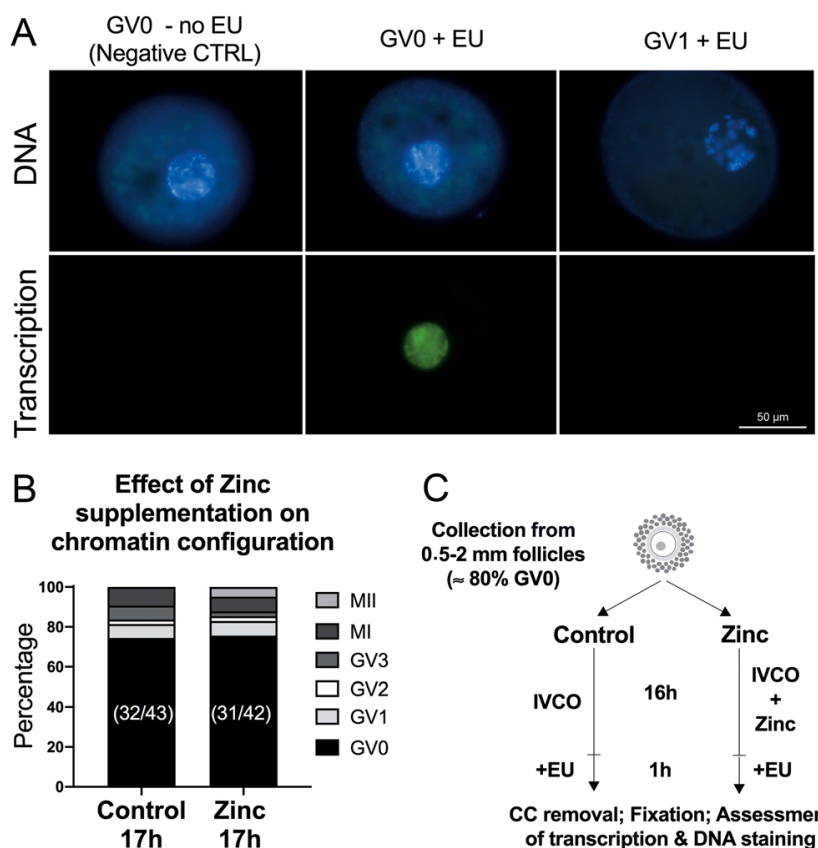

CC removal; Fixation; Assessment of transcription \& DNA staining

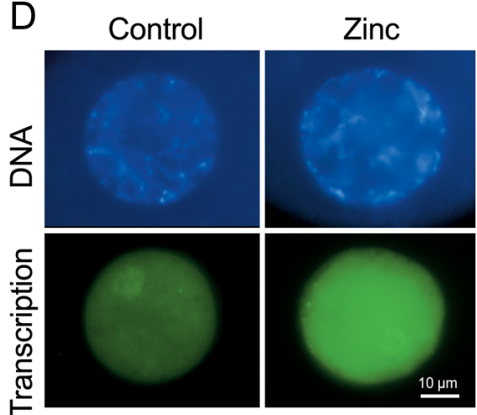

$E$

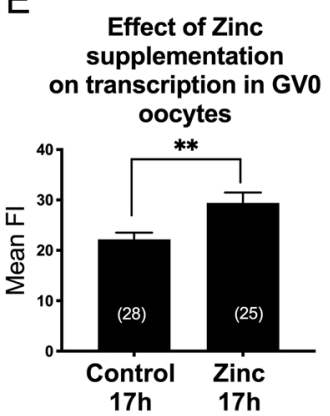

Figure 4 Effect of zinc supplementation during IVCO on global transcriptional activity in GV0 oocytes. (A) Representative images showing chromatin staining by DAPI (upper panel) and EU incorporation (lower panel) in GV0 oocytes collected from small antral follicles in the absence (negative CTRL, left panel) and presence of EU (positive CTRL, middle panel), as well as in a fully grown oocyte collected from middle antral follicle with a GV1 chromatin configuration (right panel). Note that a positive signal is detected only in GV0 oocytes in the presence of EU. (B) Schematic representation of the experimental design. (C) Graph showing the effect of zinc supplementation during $17 \mathrm{~h}$ of culture on chromatin configuration and meiotic progression. Total numbers of oocytes analyzed in two independent experiments are indicated in the graph. Note that no differences were observed. (D) Representative images showing chromatin staining by DAPI (upper panel) and transcription (lower panel) in GV0 oocytes after culture for $17 \mathrm{~h}$ under control IVCO condition or in the presence of zinc. (E) Graph showing quantification of transcriptional activity as assessed by EU incorporation into nascent RNA and fluorescent image analysis in GV0 oocytes cultured in IVCO medium in the absence or presence of zinc. Data were analyzed by unpaired $t$-test; total number of oocytes analyzed are indicated in parentheses. Values are means \pm S.E.M. ${ }^{* *}$ indicate significant differences between groups $\left({ }^{* *} P<0.01\right)$. of GV0 oocytes remained high in both control and zinc supplemented groups (Fig. 4C). Importantly, quantification of the fluorescent signal revealed that global transcription was enhanced in the zinc treated group (Fig. 4D and E).

When oocytes were exposed to the cell-permeant zinc chelator TPEN, the level of global transcription significantly decreased (Fig. 5), further supporting the hypothesis of a role of zinc in sustaining the transcriptional activity in growing oocytes. Notably, and as a control of the specificity of TPEN activity in acting as a zinc chelator, the TPEN effect was rescued by zinc supplementation, which restored the global transcription at levels comparable with the control group (Fig. 5C).

\section{Zinc supplementation affects global DNA methylation}

Notably, we did not observe gross morphological changes of the chromatin configuration after zinc supplementation under epifluorescence microscopy. Therefore, to further assess the effects of zinc supplementation on chromatin structure, we assessed the level of global DNA methylation in oocytes subjected to IVCO in the presence or absence of zinc, as DNA methylation correlates well with transcriptional activity (reviewed in Sendzikaite \& Kelsey 2019). As
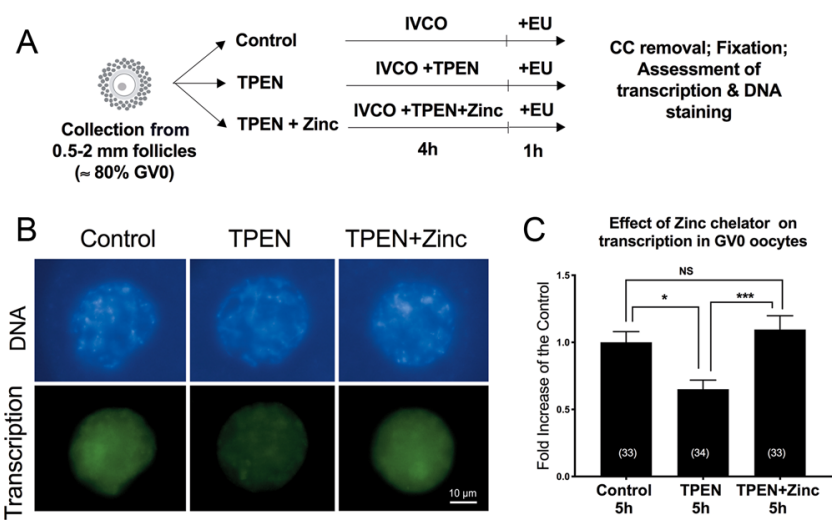

Figure 5 Effect of zinc chelation during 5-h IVCO on global transcriptional activity in GV0 oocytes. (A) Schematic representation of the experimental design. (B) Representative images showing chromatin staining by DAPI (upper panel) and transcription (lower panel) in GV0 oocytes after culture for $5 \mathrm{~h}$ under control IVCO condition, in the presence of the zinc chelator TPEN or in the presence of TPEN + zinc. (C) Graph showing quantification of transcriptional activity in GV0 oocytes cultured in IVCO medium for $5 \mathrm{~h}$ (CTRL) and in IVCO medium supplemented by TPEN or TPEN + zinc. Data were analyzed by one-way ANOVA followed by Tukey's multiple comparison test; total number of oocytes analyzed in three independent experiments are indicated in parentheses. Values are means \pm S.E.M. ${ }^{*}$ and ${ }^{* *}$ indicate significant differences between groups $(* P<0.05 ; * * P<0.01)$. 

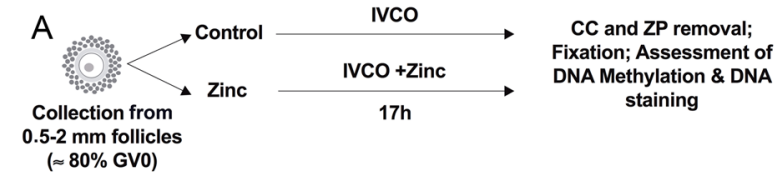

$(\approx 80 \% \mathrm{GV} 0)$
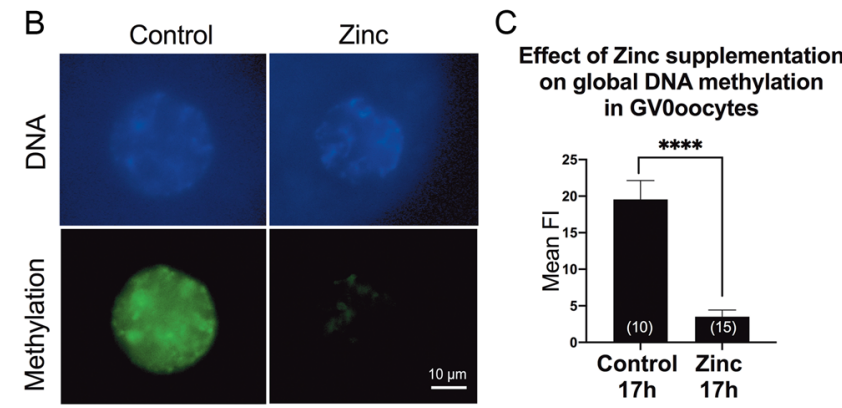

Figure 6 Effect of zinc supplementation during IVCO on global DNA methylation in GV0 oocytes. (A) Schematic representation of the experimental groups. (B) Representative images showing chromatin staining by DAPI (upper panel) and global DNA methylation (lower panel) in GV0 oocytes after culture in IVCO medium in the control (IVCO medium) and zinc (IVCO medium) in the presence of zinc groups. (C) Graph showing quantification of global DNA methylation, as assessed by indirect immunofluorescence and image analysis in GV0 oocytes cultured in IVCO medium in the absence or presence of zinc. Data were analyzed by Mann-Whitney test; total number of oocytes analyzed in two independent experiments are indicated in parentheses. Values are means \pm S.E.M. ${ }^{* * * *}$ indicate significant differences between groups $(P<0.0001)$.

shown in Fig. 6, the level of global DNA methylation is significantly lower in GVO oocytes cultured in the presence of zinc when compared to that observed in oocytes cultured in control IVCO condition. This result correlates well with higher levels of transcription in response to zinc supplementation.

\section{Discussion}

The major finding of the present study is that altering zinc availability by means of culture-medium supplementation during the final phase of their growth supports global transcriptional activity, modulates global DNA methylation and ultimately enhances acquisition of meiotic competence. Specifically, the present data indicate that the population of bovine oocytes that benefit from zinc supplementation during culture are the ones still engaged in accumulating substances for further development that are collected from early antral follicles. The final period of growth and differentiation is a crucial phase for the oocyte, as the epigenome is profoundly remodeled. Remodeling includes largescale morphological re-organization of the chromatin to form the karyosphere (Bogolyubov 2018), global transcriptional silencing as well as epigenetic modifications of DNA, such as DNA methylation, and histones proteins (De La Fuente 2006, Tomizawa et al. 2012, O'Doherty et al. 2012, Luciano \& Lodde 2013).
These modifications ultimately confer the oocyte with an appropriate developmental program.

Preliminary indication of the importance of zinc homeostasis, which in mammalian cells is performed by carrying zinc across cell compartments during oocyte growth, was revealed by the assessment of gene expression profiles of zinc transporters and methallotionein in the EmbryoGENE transcriptomic platform. To the best of our knowledge, excluding data on SLC39A6/ZIP6 and SLC39A10/ZIP10 (Kong et al. 2014) during murine oocyte maturation, no data are available on the function of other zinc transporters or MTs during mammalian oogenesis. An interesting hypothesis in the context of ovarian function is that different cellular compartments of the follicle cooperate to modulate zinc content in the follicular fluid and ultimately in the germ cell. In this view, the EmbryoGENE profiler provides interesting targets to be further functionally characterized. For instance, SLC39A8, which encodes for ZIP8, would deserve further attention, since it is differentially expressed also in granulosa cells at different stages of follicle development as reported by the EmbryoGENE profiler (http://emb-bioinfo.fsaa.ulaval.ca/IMAGE/cgibin/DoProfile.cgi?gene=slc39a8\&tissue=granulosa, July 28, 2019).

Even though the characterization of zinc transporters and MTs mRNA expression profiles is not sufficient to predict the overall zinc balance in growing oocytes, the evidences that several zinc-related transcripts are differentially expressed during this period, in both the oocyte and the surrounding cumulus cells, suggest that COCs actively control the abundance of these mRNAs, probably to modulate zinc signaling even long before meiotic resumption. This would lead to the statement that zinc availability in in vitro systems aimed to support oocyte growth has been underestimated so far. To the best of our knowledge, apart from the one developed in our laboratory and used as a base in the present study (Luciano et al. 2011), just few other studies focused on the in vitro culture of growing oocytes isolated from early antral follicles (Hirao et al. 2004, Alm et al. 2006, Endo et al. 2013, Makita \& Miyano 2014, Alam et al. 2018), while others had focused on culture of preantral oocytes. None of the published protocols can fully support embryonic developmental competence of growing oocytes. However, with the exception of serumsupplemented media, attempts to grow oocytes in vitro have been generally performed in the absence of zinc. In this study, we demonstrate that zinc supplementation to the IVCO system described by Luciano et al. (2011) benefits the acquisition of meiotic competence. This result is highly relevant in the context of modern cattle farming, which is increasingly relying on in vitro embryo production (Blondin 2015, Perry 2017). In the practice, only fully grown oocytes, which are directly subjected to IVM, are exploited. Conversely, growing oocytes contained in early antral follicles are generally discarded 
due to the lack of in vitro culture systems not being able to support their development, thus reducing the possibility of exploiting the ovarian reserve more efficiently (Lussier et al. 1987, Silva-Santos et al. 2011, Monget et al. 2012). Hence, our findings provide insights on how to improve culture media and strategies of assisted reproduction in livestock. Moreover, given the relevance of the bovine model to human ovarian physiology (Langbeen et al. 2015, Polejaeva et al. 2016), our findings would likely aid studies aimed at treating human infertility.

Defining the mechanism(s) by which zinc supports the acquisition of meiotic competence is not an easy task, due to the vast number of biological processes in which it is implicated (Beyersmann \& Haase 2001). Indeed, it has been estimated that around 2800 human proteins potentially bind zinc in vivo, which account for $10 \%$ of the human proteome (Andreini et al. 2006). Therefore, we focused our attention on one of the most important process occurring in the growing oocytes - transcription, which must be active to allow accumulation of large amounts of RNAs and proteins during growth and must be silenced timely in preparation for meiotic resumption. Zinc is a wellknown regulator, acting both as activator and repressor, of gene expression machinery (Andrews 2001, Jackson et al. 2008) and epigenetic modification, as many DNAmodifier enzymes bear zinc fingers motifs (Hudson \& Buck-Koehntop 2018, Rausch et al. 2019, Blanquart et al. 2019). Its role in controlling transcription has been considered also in the context of development of model organisms (Falchuk 1998) as well as in the mouse preimplantation embryo, where it has been shown that TPEN-induced zinc insufficiency during in vitro culture was associated with altered chromatin structure in the blastomere nuclei and decreased global transcription (Kong et al. 2015). Moreover, in the mouse model, it has been shown that transcriptionally active oocytes react to changes of zinc availability by modulating the localization of the zinc sensor MTF-1 (Kong et al. 2014), presumably to control zinc homeostasis (Andrews 2001). The present study expands these observations by showing that major transcriptional silencing in bovine oocytes, which in vivo occurs during the transition from GV0 to GV1 configuration, is associated to a decrease of labile zinc content. Accordingly, a shortterm treatment with zinc chelator TPEN induces a sudden reduction of transcription which is rescued by zinc supplementation. This might suggest that the signals that lead to transcriptional silencing in oocytes involve, at least in part, to decrease labile zinc. Interestingly, labile zinc remains relatively low in GV2 oocytes, while it increases in GV3 oocytes, in which transcription is completely silenced (Lodde et al. 2008). This agrees with the previously postulated hypothesis that increased chromatin condensation may reinforce transcriptional repression once it is initiated (De La Fuente 2006, Luciano \& Lodde 2013). If so, we can speculate that
GV3 oocytes would not be sensitive to increased labile zinc content, which in turn might correlate with increase of total zinc required during maturation in both cow and mice (Kim et al. 2010, Que et al. 2019). This hypothesis needs to be tested. Moreover, it will be of utmost importance to assess whether oocytes with different chromatin configurations vary in terms of total zinc content, as described in Que et al. (2019).

Importantly, we provide evidences that zinc supplementation enhances transcription in cultured GV0 oocytes, which well correlates with the maintenance of global level of DNA methylation that is known to characterize GV0 oocytes at the time of collection (Lodde et al. 2009). This likely accounts, at least in part, for the increased meiotic competence of zinc treated oocytes in our experimental model. Future mechanistic studies should be conducted to further clarify whether zinc supplementation affects the amount of labile and total zinc in the oocyte and, importantly, in the surrounding cumulus cells and thus clarify the molecular mechanisms through which zinc enhance oocyte capacity to undergo maturation. Nevertheless, our finding could be exploited to further ameliorate the cultural system, aiming at prolonging the period in which the oocytes remain transcriptionally active while allowing timely transcriptional silencing when the oocytes have accumulated the critical molecules for subsequent development.

\section{Conclusion}

A relationship between zinc deficiency and infertility in dairy cows has been known from many years; zinc deficiency results in increased embryonic mortality and reduced conception rates (Hidiroglou 1979). This might be due, at least in part, to the role zinc plays in oocyte development, although the mechanisms are not completely elucidated. Here we provide evidences that zinc modulates transcription during a critical step of oocyte development, which is when its developmental program is largely determined. Our results agree with previous findings in mice, where feeding a zinc deficient diet during preconception (3-5 days before ovulation) alters oocyte chromatin methylation (histone H3K4 trimethylation and global DNA methylation) and expression of specific genes (Tian \& Diaz 2013).

Clearly, it is likely that zinc participates in many other biological functions in the growing oocytes, such as mitochondrial function and metabolism regulation, and might have antioxidant/prooxidant activity (Lee 2018). Further studies are required to assess this hypothesis. Finally, of utmost importance will be the assessment of which are the relative contributions of the cumulus cells and the oocytes to the maintenance of zinc homeostasis during oogenesis as well as the characterization of specific biomarkers that primarily control importance of zinc transport and storage during oogenesis. 


\section{Supplementary materials}

This is linked to the online version of the paper at https://doi. org/10.1530/REP-19-0398.

\section{Declaration of interest}

The authors declare that there is no conflict of interest that could be perceived as prejudicing the impartiality of the research reported.

\section{Funding}

$V \mathrm{~L}$ acknowledges the receipt of a fellowship from the OECD Co-operative Research Programme: Biological Resource Management for Sustainable Agricultural Systems in 2016 (contract n. JA00091594), which supported part of this study. Part of the experiments were funded by the 'Piano Strategico Linea $\mathrm{B}^{\prime}$ of the University of Milan (PSR2019_DIP_AMLUC to A M L, PSR2018_DIP_027_VLODD to V L and PSR2018_DIP_027_ FFRAN to F F) and by PROGETTO INNOVA "PSR - Operazione 10.2.01. P C D A was supported by Scholarship from Capes foundation/PDSE/88881.134900/2016-01. EmbryoGENE Strategic Network was funded by Natural Sciences and Engineering Research Council of Canada (NSERC), Canada. C R acknowledges the Natural Sciences and Engineering Research Council of Canada (RGPIN-2017-04775).

\section{Author contribution statement}

$V L$ and $A M L$ conceived the study. C D and $V L$ participated in EmbryoGENE data analysis. M A S contributed to the study design and EmbryoGene data Analysis. R G B, V L and A M $L$ performed experiments on the assessment of the effect of zinc supplementation on meiotic competence and DNA methylation. P C D A, A B, C R and V L performed experiments on the assessment on labile zinc content in oocytes. P C D A and F F performed experiments on the assessment of transcriptional activity. $\mathrm{V} \mathrm{L}$ wrote the manuscript. All authors reviewed and approved the manuscript.

\section{Acknowledgements}

The authors are grateful to INALCA S.p.A. (Ospedaletto Lodigiano, LO) and for the support of their veterinary staff. The authors would like to thank all the scientists of the EmbryoGENE Network who contributed in creating the EmbryoGENE profiler.

\section{References}

Alam MH, Lee J \& Miyano T 2018 Inhibition of PDE3A sustains meiotic arrest and gap junction of bovine growing oocytes in in vitro growth culture. Theriogenology 118 110-118. (https://doi.org/10.1016/j. theriogenology.2018.05.028)

Alm H, Katska-Ksiazkiewicz L, Rynska B \& Tuchscherer A 2006 Survival and meiotic competence of bovine oocytes originating from early antral ovarian follicles. Theriogenology 65 1422-1434. (https://doi. org/10.1016/j.theriogenology.2005.08.014)
Anchordoquy JM, Anchordoquy JP, Sirini MA, Picco SJ, Peral-Garcia P \& Furnus CC 2014 The importance of having zinc during in vitro maturation of cattle cumulus-oocyte complex: role of cumulus cells. Reproduction in Domestic Animals 49 865-874. (https://doi.org/10.1111/rda.12385)

Andreini C, Banci L, Bertini I \& Rosato A 2006 Counting the zinc-proteins encoded in the human genome. Journal of Proteome Research 5 196-201. (https://doi.org/10.1021/pr050361j)

Andrews GK 2001 Cellular zinc sensors: MTF-1 regulation of geneexpression. Biometals 14 223-237. (https://doi.org/10.1023/a:1012932712483)

Bernhardt ML, Kim AM, O'Halloran TV \& Woodruff TK 2011 Zinc requirement during meiosis I-meiosis II transition in mouse oocytes is independent of the MOS-MAPK pathway. Biology of Reproduction $\mathbf{8 4}$ 526-536. (https://doi.org/10.1095/biolreprod.110.086488)

Bernhardt ML, Kong BY, Kim AM, O'Halloran TV \& Woodruff TK 2012 A zinc-dependent mechanism regulates meiotic progression in mammalian oocytes. Biology of Reproduction 86 114. (https://doi.org/10.1095/ biolreprod.111.097253)

Beyersmann D \& Haase H 2001 Functions of zinc in signaling, proliferation and differentiation of mammalian cells. Biometals 14 331-341. (https:// doi.org/10.1023/a:1012905406548)

Blanquart C, Linot C, Cartron PF, Tomaselli D, Mai A \& Bertrand P 2019 Epigenetic metalloenzymes. Current Medicinal Chemistry 26 2748-2785. (https://doi.org/10.2174/0929867325666180706105903)

Blondin P 2015 Status of embryo production in the world. Animal Reproduction 12 356-358.

Bogolyubov DS 2018 Karyosphere (karyosome): a peculiar structure of the oocyte nucleus. International Review of Cell and Molecular Biology 337 1-48. (https://doi.org/10.1016/bs.ircmb.2017.12.001)

De La Fuente R 2006 Chromatin modifications in the germinal vesicle (GV) of mammalian oocytes. Developmental Biology 292 1-12. (https://doi. org/10.1016/j.ydbio.2006.01.008)

Dean KM, Qin Y \& Palmer AE 2012 Visualizing metal ions in cells: an overview of analytical techniques, approaches, and probes. Biochimica and Biophysica Acta 1823 1406-1415. (https://doi.org/10.1016/j. bbamcr.2012.04.001)

Dieci C, Lodde V, Labreque R, Dufort I, Tessaro I, Sirard MA \& Luciano AM 2016 Differences in cumulus cell gene expression indicate the benefit of a pre-maturation step to improve in-vitro bovine embryo production. Molecular Human Reproduction 22 882-897. (https://doi.org/10.1093/ molehr/gaw055)

Duncan FE, Que EL, Zhang N, Feinberg EC, O'Halloran TV \& Woodruff TK 2016 The zinc spark is an inorganic signature of human egg activation. Scientific Reports 6 24737. (https://doi.org/10.1038/srep24737)

Endo M, Kawahara-Miki R, Cao F, Kimura K, Kuwayama T, Monji Y \& Iwata H 2013 Estradiol supports in vitro development of bovine early antral follicles. Reproduction 145 85-96. (https://doi.org/10.1530/REP12-0319)

Fair T, Hyttel P \& Greve T 1995 Bovine oocyte diameter in relation to maturational competence and transcriptional activity. Molecular Reproduction and Development 42 437-442. (https://doi.org/10.1002/ mrd.1080420410)

Fair T, Hyttel P, Greve T \& Boland M 1996 Nucleus structure and transcriptional activity in relation to oocyte diameter in cattle. Molecular Reproduction and Development 43 503-512. (https://doi.org/10.1002/ (SICI)1098-2795(199604)43:4<503::AID-MRD13>3.0.CO;2-\#)

Falchuk KH 1998 The molecular basis for the role of zinc in developmental biology. Molecular and Cellular Biochemistry 188 41-48. (https://doi. org/10.1023/A:1006808119862)

Hidiroglou M 1979 Trace element deficiencies and fertility in ruminants: a review. Journal of Dairy Science 62 1195-1206. (https://doi.org/10.3168/ jds.S0022-0302(79)83400-1)

Hirao Y, Itoh T, Shimizu M, Iga K, Aoyagi K, Kobayashi M, Kacchi M, Hoshi H \& Takenouchi N 2004 In vitro growth and development of bovine oocyte-granulosa cell complexes on the flat substratum: effects of high polyvinylpyrrolidone concentration in culture medium. Biology of Reproduction 70 83-91. (https://doi.org/10.1095/ biolreprod.103.021238)

Hudson NO \& Buck-Koehntop BA 2018 Zinc finger readers of methylated DNA. Molecules 23 E2555. (https://doi.org/10.3390/ molecules23102555)

Jackson KA, Valentine RA, Coneyworth LJ, Mathers JC \& Ford D 2008 Mechanisms of mammalian zinc-regulated gene expression. Biochemical 
Society Transactions 36 1262-1266. (https://doi.org/10.1042/ BST0361262)

Jeon Y, Yoon JD, Cai L, Hwang SU, Kim E, Zheng Z, Jeung E, Lee E \& Hyun SH 2015 Zinc deficiency during in vitro maturation of porcine oocytes causes meiotic block and developmental failure. Molecular Medicine Reports 12 5973-5982. (https://doi.org/10.3892/mmr.2015.4125)

Khan DR, Fournier É, Dufort I, Richard FJ, Singh J \& Sirard MA 2016 Meta-analysis of gene expression profiles in granulosa cells during folliculogenesis. Reproduction 151 R103-R110. (https://doi. org/10.1530/REP-15-0594)

Kim AM, Vogt S, O'Halloran TV \& Woodruff TK 2010 Zinc availability regulates exit from meiosis in maturing mammalian oocytes. Nature Chemical Biology 6 674-681. (https://doi.org/10.1038/nchembio.419)

Kim AM, Bernhardt ML, Kong BY, Ahn RW, Vogt S, Woodruff TK \& O'Halloran TV 2011 Zinc sparks are triggered by fertilization and facilitate cell cycle resumption in mammalian eggs. ACS Chemical Biology 6 716-723. (https://doi.org/10.1021/cb200084y)

Kimura T \& Kambe T 2016 The functions of metallothionein and ZIP and $\mathrm{ZnT}$ transporters: an overview and perspective. International Journal of Molecular Sciences 17 336. (https://doi.org/10.3390/ijms17030336)

Kong BY, Bernhardt ML, Kim AM, O'Halloran TV \& Woodruff TK 2012 Zinc maintains prophase I arrest in mouse oocytes through regulation of the MOS-MAPK pathway. Biology of Reproduction 87 11, 1-12. (https:// doi.org/10.1095/biolreprod.112.099390)

Kong BY, Duncan FE, Que EL, Kim AM, O'Halloran TV \& Woodruff TK 2014 Maternally-derived zinc transporters ZIP6 and ZIP10 drive the mammalian oocyte-to-egg transition. Molecular Human Reproduction 20 1077-1089. (https://doi.org/10.1093/molehr/gau066)

Kong BY, Duncan FE, Que EL, Xu Y, Vogt S, O'Halloran TV \& Woodruff TK 2015 The inorganic anatomy of the mammalian preimplantation embryo and the requirement of zinc during the first mitotic divisions. Developmental Dynamics 244 935-947. (https://doi.org/10.1002/ dvdy.24285)

Labrecque R, Lodde V, Dieci C, Tessaro I, Luciano AM \& Sirard MA 2015 Chromatin remodelling and histone $\mathrm{m}$ RNA accumulation in bovine germinal vesicle oocytes. Molecular Reproduction and Development 82 450-462. (https://doi.org/10.1002/mrd.22494)

Labrecque R, Fournier E \& Sirard MA 2016 Transcriptome analysis of bovine oocytes from distinct follicle sizes: insights from correlation network analysis. Molecular Reproduction and Development 83 558-569. (https://doi.org/10.1002/mrd.22651)

Langbeen A, de porte HF, Bartholomeus E, Leroy JL \& Bols PE 2015 Bovine in vitro reproduction models can contribute to the development of (female) fertility preservation strategies. Theriogenology 84 477-489. (https://doi.org/10.1016/j.theriogenology.2015.04.009)

Lee SR 2018 Critical role of zinc as either an antioxidant or a prooxidant in cellular systems. Oxidative Medicine and Cellular Longevity 2018 9156285. (https://doi.org/10.1155/2018/9156285)

Lisle RS, Anthony K, Randall MA \& Diaz FJ 2013 Oocyte-cumulus cell interactions regulate free intracellular zinc in mouse oocytes. Reproduction 145 381-390. (https://doi.org/10.1530/REP-12-0338)

Lodde V, Modina S, Galbusera C, Franciosi F \& Luciano AM 2007 Largescale chromatin remodeling in germinal vesicle bovine oocytes: interplay with gap junction functionality and developmental competence. Molecular Reproduction and Development 74 740-749. (https://doi. org/10.1002/mrd.20639)

Lodde V, Modina S, Maddox-Hyttel P, Franciosi F, Lauria A \& Luciano AM 2008 Oocyte morphology and transcriptional silencing in relation to chromatin remodeling during the final phases of bovine oocyte growth. Molecular Reproduction and Development 75 915-924. (https://doi. org/10.1002/mrd.20824)

Lodde V, Modina SC, Franciosi F, Zuccari E, Tessaro I \& Luciano AM 2009 Localization of DNA methyltransferase-1 during oocyte differentiation, in vitro maturation and early embryonic development in cow. European Journal of Histochemistry 53 e24. (https://doi.org/10.4081/ejh.2009.e24)

Luciano AM \& Lodde V 2013 Changes of large-scale chromatin configuration During mammalian oocyte differentiation. In Oogenesis. G Coticchio, DF Albertini \& L De Santis. London: Springer. (https://doi. org/10.1007/978-0-85729-826-3)

Luciano AM, Franciosi F, Modina SC \& lodde V 2011 Gap junctionmediated communications regulate chromatin remodeling during bovine oocyte growth and differentiation through cAMP-dependent mechanism(s). Biology of Reproduction 85 1252-1259. (https://doi. org/10.1095/biolreprod.111.092858)

Luciano AM, Franciosi F, Dieci C \& Lodde V 2014a Changes in largescale chromatin structure and function during oogenesis: a journey in company with follicular cells. Animal Reproduction Science 149 3-10. (https://doi.org/10.1016/j.anireprosci.2014.06.026)

Luciano AM, Franciosi F, Dieci C, Tessaro I, Terzaghi L, Modina SC \& Lodde V 2014b Large-scale chromatin structure and function changes during oogenesis: the interplay between oocyte and companion cumulus cells. Animal Reproduction 11 141-149.

Lussier JG, Matton P \& Dufour JJ 1987 Growth rates of follicles in the ovary of the cow. Journal of Reproduction and Fertility 81 301-307. (https://doi.org/10.1530/jrf.0.0810301)

Makita M \& Miyano T 2014 Steroid hormones promote bovine oocyte growth and connection with granulosa cells. Theriogenology 82 605-612. (https://doi.org/10.1016/j.theriogenology.2014.05.020)

Maret W 2017 Zinc in cellular regulation: the nature and significance of 'zinc signals'. International Journal of Molecular Sciences 18 E2285. (https://doi.org/10.3390/ijms18112285)

Monget P, Bobe J, Gougeon A, Fabre S, Monniaux D \& Dalbies-Tran R 2012 The ovarian reserve in mammals: a functional and evolutionary perspective. Molecular and Cellular Endocrinology 356 2-12. (https:// doi.org/10.1016/j.mce.2011.07.046)

O'Doherty AM, O'Shea LC \& Fair T 2012 Bovine DNA methylation imprints are established in an oocyte size-specific manner, which are coordinated with the expression of the DNMT3 family proteins. Biology of Reproduction 86 67. (https://doi.org/10.1095/biolreprod.111.094946)

Outten CE \& O'Halloran TV 2001 Femtomolar sensitivity of metalloregulatory proteins controlling zinc homeostasis. Science $\mathbf{2 9 2}$ 2488-2492. (https://doi.org/10.1126/science.1060331)

Perry AC 20172016 Statistics of embryo collection and transfer in domestic farm animals. (available at: https://www.iets.org/comm_data.asp?autotry $=$ true $\& U L$ notkn=true)

Picco SJ, Anchordoquy JM, De Matos DG, Anchordoquy JP, Seoane A, Mattioli GA, Errecalde AL \& Furnus CC 2010 Effect of increasing zinc sulphate concentration during in vitro maturation of bovine oocytes. Theriogenology 74 1141-1148. (https://doi.org/10.1016/j. theriogenology.2010.05.015)

Polejaeva IA, Rutigliano HM \& Wells KD 2016 Livestock in biomedical research: history, current status and future prospective. Reproduction, Fertility, and Development 28 112-124. (https://doi.org/10.1071/ RD15343)

Que EL, Bleher R, Duncan FE, Kong BY, Gleber SC, Vogt S, Chen S, Garwin SA, Bayer AR, Dravid VP et al. 2015 Quantitative mapping of zinc fluxes in the mammalian egg reveals the origin of fertilizationinduced zinc sparks. Nature Chemistry 7 130-139. (https://doi. org/10.1038/nchem.2133)

Que EL, Duncan FE, Bayer AR, Philips SJ, Roth EW, Bleher R, Gleber SC, Vogt S, Woodruff TK \& O'Halloran TV 2017 Zinc sparks induce physiochemical changes in the egg zona pellucida that prevent polyspermy. Integrative Biology: Quantitative Biosciences from Nano to Macro 9 135-144. (https://doi.org/10.1039/c6ib00212a)

Que EL, Duncan FE, Lee HC, Hornick JE, Vogt S, Fissore RA, O'Halloran TV \& Woodruff TK 2019 Bovine eggs release zinc in response to parthenogenetic and sperm-induced egg activation. Theriogenology 127 41-48. (https://doi.org/10.1016/j.theriogenology.2018.12.031)

Rausch C, Hastert FD \& Cardoso MC 2019 DNA modification readers and writers and their interplay. Journal of Molecular Biology 432 1731-1746. (https://doi.org/10.1016/j.jmb.2019.12.018)

Sanchez F, Romero S, De Vos M, Verheyen G \& Smitz J 2015 Human cumulus-enclosed germinal vesicle oocytes from early antral follicles reveal heterogeneous cellular and molecular features associated with in vitro maturation capacity. Human Reproduction 30 1396-1409. (https:// doi.org/10.1093/humrep/dev083)

Schneider CA, Rasband WS \& Eliceiri KW 2012 NIH Image to Image): 25 years of image analysis. Nature Methods 9 671-675. (https://doi. org/10.1038/nmeth.2089)

Sendzikaite G \& Kelsey G 2019 The role and mechanisms of DNA methylation in the oocyte. Essays in Biochemistry 63 691-705. (https:// doi.org/10.1042/EBC20190043)

Silva-Santos KC, Santos GM, Siloto LS, Hertel MF, Andrade ER, Rubin MI, Sturion L, Melo-Sterza FA \& Seneda MM 2011 Estimate of the population 
of preantral follicles in the ovaries of Bos taurus indicus and Bos taurus taurus cattle. Theriogenology 76 1051-1057. (https://doi.org/10.1016/j. theriogenology.2011.05.008)

Tian X \& Diaz FJ 2012 Zinc depletion causes multiple defects in ovarian function during the periovulatory period in mice. Endocrinology 153 873-886. (https://doi.org/10.1210/en.2011-1599)

Tian X \& Diaz FJ 2013 Acute dietary zinc deficiency before conception compromises oocyte epigenetic programming and disrupts embryonic development. Developmental Biology 376 51-61. (https://doi. org/10.1016/j.ydbio.2013.01.015)

Tian X, Anthony K, Neuberger T \& Diaz FJ 2014 Preconception zinc deficiency disrupts postimplantation fetal and placental development in mice. Biology of Reproduction 90 83. (https://doi.org/10.1095/ biolreprod.113.113910)

Tomizawa S, Nowacka-Woszuk J \& Kelsey G 2012 DNA methylation establishment during oocyte growth: mechanisms and significance. International Journal of Developmental Biology 56 867-875. (https://doi. org/10.1387/ijdb.120152gk)
Zhao MH, Kwon JW, Liang S, Kim SH, Li YH, Oh JS, Kim NH \& Cui XS 2014 Zinc regulates meiotic resumption in porcine oocytes via a protein kinase C-related pathway. PLoS ONE 9 e102097. (https://doi. org/10.1371/journal.pone.0102097)

Zuccotti M, Garagna S, Merico V, Monti M \& Alberto Redi C 2005 Chromatin organisation and nuclear architecture in growing mouse oocytes. Molecular and Cellular Endocrinology 234 11-17. (https://doi. org/10.1016/j.mce.2004.08.014)

Received 27 August 2019

First decision 27 September 2019

Revised manuscript received 25 February 2020

Accepted 19 March 2020 